\title{
A FORMAÇÃO DA PERCEPÇÃO DE VALOR PARA PEQUENOS E MÉDIOS CONSUMIDORES B2B
}

Objetivo: identificar e mensurar as relações entre constructos de qualidade-sacrifícios-experiência e valor percebido e satisfação, e a relação destes com a intenção de recompra de serviços logísticos de transporte por PMEs.

Método: Partindo-se da proposição de escalas para mensurar os constructos qualidade, sacrifícios, experiência, valor percebido, satisfação e intenção de recompra, realizou-se um levantamento de dados cuja amostra foi composta por 195 PMEs. Utilizando técnicas de AFE, AFC e MEEPLS, propôs-se um modelo para mensurar a formação do valor percebido nos serviços logísticos adquiridos por PMEs.

Originalidade/Relevância: A maioria das pesquisas de valor percebido no ambiente B2B tem considerado o desempenho dos bens e serviços em uma perspectiva funcional e negligenciado dimensões não-racionais. Estas dimensões (aspectos não-econômicos, subjetivos e multidimensionais), se mensuradas em conjunto com dimensões comumente encontradas nos estudos de comportamento de consumo B2B, podem oferecer novas perspectivas de conhecimento teórico-empírico.

Resultados: Os sacrifícios e os custos de troca atuaram de forma não-significante no relacionamento B2B. As PMEs valorizaram aspectos ligados à qualidade e à experiência. O estudo indica que PMEs, ao elegerem como formadores do valor percebido a experiência e a qualidade, e por serem influenciadas pela satisfação para formar suas intenções de recompras, gerenciam sua cadeia de suprimentos estabelecendo relacionamentos envoltos em mais subjetividades do que se esperaria de um empresário.

Contribuições teóricas/metodológicas: Proposição de um modelo teórico capaz de predizer a intenção de recompra de PMEs consumidoras de serviços logísticos, tendo como antecessor a satisfação, e desta, o valor percebido.

Palavras-chave: Valor Percebido. Experiência. Satisfação. Intenção de Recompra. Marketing B2B. 


\section{THE FORMATION OF THE PERCEPTION OF VALUE FOR SMALL AND MEDIUM B2B CONSUMERS}

Objective: To identify and measure the relationships between quality-sacrifice-experience constructs and perceived value and satisfaction, and their relationship with the intention of repurchasing transportation logistics services by SMEs.

Method: Starting from the proposition of scales to measure the constructs quality, sacrifices, experience, perceived value, satisfaction and intention to repurchase, a data survey was carried out, whose sample was composed of 195 SMEs. Using EFA techniques, CFA and MEEPLS, a model was proposed to measure the formation of perceived value in logistics services acquired by SMEs.

Originality / Relevance: Most perceived value surveys in the B2B environment have considered the performance of goods and services in a functional perspective and overlooked non-rational dimensions. These dimensions (noneconomic, subjective, and multidimensional aspects), if measured in conjunction with dimensions commonly encountered in B2B consumer behavior studies, may offer new perspectives of theoretical-empirical knowledge.

Results: The sacrifices and exchange costs were non-significant in the B2B relationship. SMEs have valued aspects related to quality and experience. The study indicates that SMEs, by choosing as perceived value trainers experience and quality, and because they are influenced by satisfaction to form their repurchase intentions, manage their supply chain by establishing relationships wrapped in more subjectivities than one would expect from an entrepreneur.

Theoretical / methodological contributions: Proposition of a theoretical model capable of predicting the repurchase intention of SMEs consuming logistic services, having as its predecessor the satisfaction, and as its own, the perceived value.

Keywords: Perceived Value. Experience. Satisfaction. Repurchase Intention. B2B Marketing.

Clarice Mara Sousa-e-Silva ${ }^{1}$

Stella Naomi Moriguchi ${ }^{2}$ José Eduardo Ferreira Lopes ${ }^{3}$

\footnotetext{
${ }^{1}$ Mestre em Administração pela Universidade Federal de Uberlândia - UFU. Minas Gerais, Brasil. E-mail: claricemara@ hotmail.com

2 Doutora em Administração pela Universidade de São Paulo - USP. São Paulo, Brasil. E-mail: stellanaomi@gmail.com

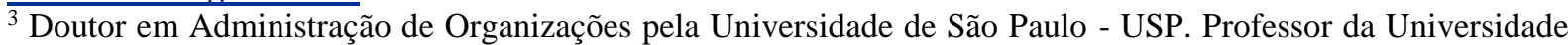
Federal de Uberlândia - UFU. Minas Gerais, Brasil. E-mail: jeflopes@fagen.ufu.br
} 


\section{INTRODUÇÃO}

A questão ligada ao que seja valor, como deve ser capturado, construído e entregue ao cliente constitui-se em fonte de preocupações tanto de acadêmicos quanto de profissionais de marketing (Varadarajan, 2010). Dentre as diversas abordagens do que seja valor para clientes, encontra-se aquela que se refere ao valor percebido: avaliação geral que o consumidor faz sobre a utilidade de um bem ou serviço, baseando-se em percepções do que recebe (benefícios), comparando-o com os sacrifícios que precisa fazer para obtê-los (Zeithaml, 1988).

No ambiente de trocas em mercados organizacionais a formação do valor percebido precisa ser investigada de forma distinta das trocas realizadas em mercados de consumo (Anderson \& Narus, 1999). No que tange à investigação do valor percebido em mercados B2B, Lindgreen, Hingley, Grant \& Morgan (2012) propõem um aprofundamento na compreensão de como este é analisado, criado e entregue. Postulam também que métricas de valor são importantes para futuras pesquisas, e que as empresas devem continuar a desenvolver, monitorar e avaliar tais métricas (Lindgreen et al., 2012). Seja no ambiente de serviços B2B ou B2C, pesquisadores sugerem que métricas de valor precisam considerar $\mathrm{O}$ desenvolvimento de uma articulação mais clara entre os constructos ligados ao valor percebido, à qualidade do serviço, preço, os benefícios, os sacrifícios, à satisfação do cliente e, finalmente, intenções comportamentais (Boksberger \& Melsen, 2011).

No Brasil, a pesquisa de valor percebido apresenta-se principalmente focada em relações B2C, e investigações do ambiente B2B são escassas no país (Lacerda \& Mendonça, 2010; Rocha \& Silva, 2006). Já, no âmbito internacional, pesquisas na área de percepção de valor no ambiente B2B têm sido publicadas e clarificam o fenômeno (Eggert \& Ulaga, 2002; Fiol, Tena \& García, 2011; Lam, Shankar, Erramilli \& Murthy, 2004; Molinari, Abratt \& Dion, 2008). A necessidade de investigação na área de valor, aprofundando o conhecimento dos atributos formadores do valor percebido e suas consequentes intenções comportamentais de compra (Boksberger \& Melsen, 2011; Lindgreen et al., 2012), é o que motiva as investigações deste trabalho.

No ambiente de prestação de serviços, tem se destacado no Brasil atualmente um mercado de serviços logísticos complexo (Confederação Nacional dos Transportes [CNT], 2014), caracterizado por demandantes que necessitam cruzar um país continental com as suas mercadorias, e ofertantes que crescem e se diferenciam para atender a demandas cada vez mais específicas
(Figueiredo \& Mora, 2009). Tradicionalmente, por não fazerem parte das competências essenciais das empresas, os serviços logísticos, em especial os de armazenagem, movimentação e transporte, costumam ser candidatos à terceirização, o que propicia o surgimento de um mercado concorrencial de prestadores destes serviços (Figueiredo \& Mora, 2009).

Dentre os diversos tipos de serviços logísticos terceirizados neste mercado, importante destaque dá-se ao transporte rodoviário de cargas (TRC). Atualmente, o Brasil possui mais de um milhão de empresas prestadoras deste tipo de serviço registradas na Agência Nacional de Trânsito e Transportes, entre autônomos, transportadores e cooperativas, e quase dois milhões de veículos de TRC (http://www.antt.gov.br/, recuperado em 04, março, 2017). Além disto, o TRC é o modal de maior representatividade no país: mais de $60 \%$ da carga movimentada no Brasil utiliza este modal (CNT, 2014).

Devido à complexidade e continuidade envolvidas na prestação de serviços logísticos de transporte, estes constituem um importante mercado a ser investigado pelo marketing (Lam et al., 2004; Molinari et al., 2008). A gestão da cadeia de suprimentos e a avaliação de desempenho de fornecedores tem sido tarefa problemática para as empresas como um todo, incluindo os serviços logísticos, que se constituem em um desafio tanto para pequenas/médias como para as grandes corporações (Hong \& Jeong, 2006; Pressey, Winklhofer \& Tzokas, 2009; Ozmen, Oner, Khosrowshahi, \& Underwood, 2013).

Quando se trata de Pequenas e Médias Empresas (PMEs), as dificuldades na gestão de transportes terceirizados podem se agravar, pois estas atuam em contextos distintos das grandes corporações (Hong \& Jeong, 2006; Ozmen et al., 2013). Pressey et al. (2009) mostraram que a avaliação dos fornecedores pelas PMEs obtêm resultados inferiores às daquelas praticadas pelas grandes corporações, devido à escassez de tempo e recursos que enfrentam. No entanto, é comum que tal lacuna de mensuração objetiva de desempenho seja suprida com o desenvolvimento de relacionamentos cooperativos e/ou baseados na personalidade do dono da PME, ou mesmo priorizando fornecedores mais estáveis que possam minimizar perdas. Os desafios enfrentados na gestão de PMEs envolvem escassez de recursos financeiros, pessoas, sistemas e conhecimento técnico (Drumond \& Toaldo, 2009). Além disto, a gestão de pequenas e médias empresas tem forte componente familiar e centralização na gestão, e o dono acaba por envolver-se nas decisões e atividades funcionais, muitas vezes de maneira não planejada, impactando 
negativamente em seus resultados (Drumond \& Toaldo, 2009; Serviço Brasileiro de Apoio às Micro e Pequenas Empresas [SEBRAE], 2013).

Dados os altos custos logísticos de transporte envolvidos (CNT, 2014), complexidades inerentes ao processo de gestão de transportes (Hong \& Jeong, 2006) e peculiaridades ligadas ao perfil dos pequenos e médios empreendedores (Pressey et al., 2009; Ozmen et al., 2013; SEBRAE, 2013), torna-se relevante compreender mais profundamente como se formam suas percepções de valor quando consomem e se relacionam com seus parceiros logísticos.

Tradicionalmente, as decisões de compradores no ambiente B2B são tomadas levando-se em consideração o desempenho dos bens e serviços em uma perspectiva funcional, e a maioria das pesquisas de valor percebido no ambiente B2B tem negligenciado dimensões não-racionais. Por isto, estudos recentes propõem que os modelos predominantemente utilitários e objetivos de mensuração de valor no ambiente B2B considerem também aspectos não-econômicos, subjetivos $\mathrm{e}$ multidimensionais, incluindo por exemplo o papel das emoções, do relacionamento e da experiência no comportamento de compra organizacional. (Mencarelli \& Rivière, 2015). Tais dimensões nãoracionais, se mensuradas em conjunto com dimensões comumente encontradas nos estudos de comportamento de consumo B2B podem oferecer novas perspectivas de conhecimento teóricoempírico.

Desta forma, a presente pesquisa objetivou identificar e mensurar as relações entre constructos de qualidade-sacrifícios-experiência e valor percebido e satisfação, e a relação destes com a intenção de recompra de serviços logísticos de transporte por PMEs, considerando os constructos experiência e satisfação como dimensões nãoracionais que podem ser encontradas não apenas no ambiente B2C, mas também no B2B. Destaca-se que a abordagem de experiência e satisfação em uma perspectiva não-racional no contexto $\mathrm{B} 2 \mathrm{~B}$ sugere novas perspectivas para a compreensão do comportamento B2B no Brasil.

$\mathrm{Na}$ próxima seção revisa-se trabalhos teóricos e empíricos relacionados ao valor percebido e suas relações com a qualidade, os sacrifícios, a experiência, a satisfação e a intenção de recompra.

\section{REVISÃO TEÓRICA E EMPÍRICA}

\subsection{Valor Percebido, a Qualidade e os Sacrifícios}

A definição de valor apresenta diversas abordagens. Dentre tais conceitos, encontra-se a perspectiva adotada nesta pesquisa: o valor percebido pelo cliente. No Brasil, o termo é conhecido como valor percebido ou valor para o cliente, pois utiliza o ponto de vista do cliente nos julgamentos de valor (Mello \& Leão, 2008), e pode ser considerado como uma relação entre benefícios recebidos e sacrifícios empenhados para consumir um determinado bem ou serviço.

Mensurar valor percebido é tarefa que precisa considerar sua multidimensionalidade e complexidade, levando-se em consideração dimensões de qualidade, sacrifícios e experiências vividas no uso do produto (Ravald \& Grönroos, 1996). A literatura de marketing tem considerado que os benefícios recebidos em uma situação de consumo constituem a primeira dimensão da avaliação de um consumidor para formar sua percepção de valor. Cronin Jr., Brady e Hult (2000), Fornell, Johnson, Anderson, Cha \& Bryant (1996) e Zeithaml (1988) consideram que benefícios recebidos pelos consumidores podem ser traduzidos em qualidade percebida. Este conceito vem à tona para evidenciar que altos níveis de abstração realizados em avaliações podem levar em conta o desempenho de atributos, mas estes por si só não conseguem formar a percepção de qualidade. A qualidade percebida é um julgamento atitudinal de valor, que avalia a superioridade ou excelência de um produto, onde se evoca um grupo de produtos similares que são postos em comparação (Zeithaml, 1988).

Para testar a relação entre qualidade percebida e valor percebido, a presente pesquisa propôs a primeira hipótese $(\mathrm{H} 1)$ - "A qualidade percebida influencia positivamente o valor percebido", e a segunda hipótese (H2) - "A qualidade percebida influencia positivamente a satisfação" utilizando a perspectiva de Parasuraman, Zeithaml \& Berry (1988) de confiabilidade no constructo "Qualidade do Serviço".

Já no que se refere aos sacrifícios, estes podem ser considerados como aquilo que é dado para adquirir um serviço (Cronin Jr et al., 2000). Zeithaml (1988) propõe que o preço monetário é um importante componente dos sacrifícios, mas não é o único, e ainda sugere que os componentes do sacrifício no valor percebido incluem preços monetários (custos objetivos) e não-monetários (tempo, energia, esforços). Uma abordagem complementar de sacrifícios encontrada na literatura B2B são os custos de troca, sugerindo que nos relacionamentos cliente-fornecedor, o sacrifício empenhado em uma possível mudança de fornecedor pode envolver desgastes e prejuízos significantes, inibindo uma possível alteração de fornecedor (Fiol et al., 2011; Lam et al., 2004).

Os custos de troca podem ser definidos como os custos que os clientes associam com o processo de mudança de um fornecedor para outro 
(Burnham, Frels \& Mahajan, 2003), ou como a avaliação de troca de fornecedor em que a alternativa possa ser segura e viável (Yanamandram \& White, 2006). Patterson (2004) identificou correlações relevantes entre satisfação e retenção de clientes, quando os custos de troca são baixos. Tal pesquisa pode colaborar com a compreensão de que, quando barreiras de troca são percebidas como baixas, consumidores estão livres para trocarem de fornecedor caso estejam insatisfeitos, ou manterem uma relação com o atual fornecedor caso estejam satisfeitos. No ambiente $\mathrm{B} 2 \mathrm{~B}$, a mesma relação é sugerida por Yanamandram e White (2006). Para estes autores, muitos clientes do ambiente B2B podem estar mantendo relacionamentos com seus fornecedores não porque estejam satisfeitos, mas porque encontram custos de troca muito altos.

Para testar a relação entre os sacrifícios e o valor percebido, considerou-se o constructo "Sacrifícios" com formado por custos monetários, custos não-monetários e custos de troca, propondose as duas hipóteses seguintes: H3 - "Os sacrifícios influenciam negativamente o valor percebido", conforme Oliver (1994) e Patterson (2004) e H4 "Os sacrifícios influenciam negativamente a satisfação", baseando-se em Bitner, Booms e Tetreault (1990), Lemke et al. (2011) e Oliver (1994).

\subsection{O Valor Percebido e a Experiência}

O estudo de Flint, Woodruff, \& Gardial (1997), ao definir os conceitos de valores, valores desejados e valores de julgamento, sustenta que as avaliações do consumidor podem depender do uso, sugerindo que a experiência é um fator importante na formação da percepção de valor. Ravald e Grönroos (1996) também corroboram a importância da experiência na formação da percepção de valor e propõem que, além desta comparação entre benefícios e sacrifícios, é preciso considerar que o consumo ocorre em diversos episódios que darão ao consumidor sua perspectiva de valor.

Estudos sobre experiência em serviços ligados ao ambiente $\mathrm{B} 2 \mathrm{~B}$ vieram à tona realizando adaptações do ambiente B2C para este contexto específico (Irene, Berenguer \& Cervera, 2008; Johnston \& Kong, 2011). Para Lemke, Clark e Wilson (2011), tanto em ambientes B2C como em $\mathrm{B} 2 \mathrm{~B}$, consumidores podem articular um complexo grupo de constructos ligados à comunicação, encontros de uso e serviços, por meio dos quais julgam a excelência ou a superioridade de suas experiências.

Os achados de pesquisas ligadas ao ambiente B2B consideram essencialmente que, por meio da experiência vivenciada na prestação dos serviços, o consumidor forma suas percepções de valor e os encontros de serviços podem ser considerados como um importante antecedente da avaliação que os consumidores fazem da performance de serviços (Irene et al., 2008).

No ambiente B2B, aspectos ligados à experiência têm sido associados predominantemente ao relacionamento estabelecido entre o cliente e o fornecedor (Fiol et al., 2011; Johnston \& Kong, 2011; Lemke et al., 2011). Sob a perspectiva destes autores e de Parasuraman et al. (1988), a presente pesquisa selecionou cinco aspectos relevantes na avaliação da experiência em serviços logísticos para formar o constructo "Experiência": (1) Relacionamento Interpessoal, (2) Flexibilidade, (3) Empatia, (4) Credibilidade e (5) Conhecimento Técnico, propondo a quinta hipótese (H5) - "As experiências influenciam positivamente $\mathrm{o}$ valor percebido" e a sexta hipótese (H6) - "As experiências influenciam positivamente a satisfação".

\subsection{O Valor Percebido, a Satisfação e a Intenção de Recompra}

Considerada um melhor preditor de intenções comportamentais do que o valor percebido (Chaudhuri, 2006), a satisfação tem sido investigada como uma consequência do valor percebido (Eggert \& Ulaga, 2002; Fornell et al., 1996). Oliver (2006) conceitua satisfação como um juízo que um indivíduo faz de uma característica do produto ou do produto em si, advindo de um nível prazeroso de cumprimento relacionado ao consumo, incluindo os níveis de cumprimento aquém ou além das expectativas.

Mesmo considerando-se a importância de se investigar a satisfação em ambiente B2B, a relevância do valor percebido em detrimento de métricas de satisfação ancora-se também no fato de que, mesmo satisfeitos, clientes podem deixar de comprar um produto (Ulaga \& Chacour, 2001). Por este motivo, pesquisas no ambiente B2B investigam valor percebido como um constructo distinto da satisfação (Eggert \& Ulaga, 2002). Baseando-se na concepção de Oliver (1996), autores como Eggert e Ulaga (2002) consideraram que a satisfação seria uma resposta afetiva à percepção do valor, e procuraram mensurar variáveis ligadas ao prazer de se ter uma relação de compra com o fornecedor e à possibilidade de o cliente ter encontrado o fornecedor ideal.

Para se analisar a relação entre valor percebido e satisfação, propõe-se a sétima hipótese, cuja investigação ancora-se em estudos como os de Blocker (2011), Eggert e Ulaga (2002), Lam et al. (2004), McDougall e Levesque (2000) e Spiteri e Dion (2004): H7 - "O valor percebido influencia positivamente a satisfação”. Tais estudos revelam 
que a satisfação pode ter efeitos diferentes em contextos diferentes quando relacionada ao valor percebido, evidenciando que a ligação entre satisfação e valor percebido não está totalmente clarificada.

Além disto, valor percebido e satisfação atuam de formas diferentes na intenção de recompra. Eggert e Ulaga (2002), por exemplo, sugerem que a satisfação possui uma carga emocional mais presente que o valor percebido na formação da intenção de recompra. Já Oliver (1994), em sua pesquisa ligada às emoções, satisfação e qualidade em um ambiente de consumo de serviços, propõe que tanto variáveis cognitivas quanto afetivas contribuem de maneira significativa na construção do que seja satisfação. Este autor ressalta que, aparentemente, dois mecanismos operam em conjunto nas mentes dos consumidores: um envolve a avaliação comparativa de resultados funcionais (o que o serviço me deu), e outro relacionado a como o serviço influencia o afeto (como o serviço fez com que eu me sentisse) (Oliver, 1994). A mesma abordagem pode ser vista em Chaudhuri (2006), o qual sugere que, apesar de afetos não dependerem de cognições racionais, todas as cognições racionais são acompanhadas de afeto. Devido a este contexto de complexidades racionais e não-racionais, torna-se importante que tanto satisfação quanto valor percebido sejam investigados em suas relações com a intenção de recompra.

A intenção de recompra figura como uma importante intenção comportamental de consumidores, pois por meio dela pode-se relacionar os esforços empreendidos por uma empresa em prestar um serviço de qualidade que gere impacto financeiro de tais investimentos (Zeithaml, Berry \& Parasuraman, 1996). É também uma das diversas intenções comportamentais que um consumidor pode ter em um processo de consumo (Fishbein \& Ajzen, 1975).

Pesquisadores consideram, sob a ótica de Fishbein e Azjen (1975), que valor percebido possui alta carga de cognição, por ser um constructo eminentemente avaliativo e racional (Eggert \& Ulaga, 2002; Ulaga \& Chacour, 2001). Como consequência do valor percebido, a satisfação pode ser considerada uma dimensão com forte conotação emocional e atitudinal (Lam et al., 2004), e as intenções comportamentais podem ser consideradas como influenciadas pela satisfação (Eggert \& Ulaga, 2002).

A intenção de recompra pode ser diretamente influenciada tanto pela satisfação (Patterson, 2004; Kumar, 2002) como também pelo valor percebido, sustentando-se na premissa de que modelos deliberativos como o de Fishbein e Azjen (1975) sugerem que um indivíduo processa informações, forma crenças, pesa prós e contras antes de decidir, baseando-se em julgamentos conscientes (Bagozzi, Gürhan-Canli \& Priester, 2002). Assim o presente estudo propõe três hipóteses para clarificar o fenômeno: $\mathrm{H} 7$ - "O valor percebido influencia positivamente a satisfação" (Blocker, 2011; Eggert \& Ulaga,2002; Lam et al., 2004; McDougall \& Levesque, 2000; Spiteri \& Dion, 2004), H8 - “A satisfação influencia positivamente a intenção de recompra" (Fishbein \& Azjen, 1975; Cronin et al., 2000; Eggert \& Ulaga, 2002; Kumar, 2002; Patterson, 2004), e H9 - "O valor percebido influencia positivamente a intenção de recompra" (Cronin et al., 2000; Eggert \& Ulaga, 2002; Molinari et al.,2008).

Para testar a hipóteses apresentadas, realizou-se um levantamento, a seguir detalhado juntamente com as técnicas utilizadas para análise dos dados posteriormente apresentada.

\section{ASPECTOS METODOLÓGICOS}

A presente pesquisa pode ser caracterizada como aplicada, empírica, descritiva e hipotéticodedutiva (Lakatos \& Marconi, 2000). Quanto ao procedimento técnico, realizou-se um levantamento quantitativo-descritivo (Gil, 2002). O instrumento de coleta de dados proposto foi adaptado de escalas já testadas e publicadas: Parasauraman et al. (1988), Fiol et al. (2011), Eggert e Ulaga (2002) Fornell et al. (1996); Lam et al. (2004), Molinari, Abratt e Dion (2008) e Lapierre (2000), adequando-as aos objetivos desta pesquisa. O Quadro 1 apresenta as variáveis do instrumento de coleta de coleta de dados. 


\begin{tabular}{|c|c|c|}
\hline Constructo & \multicolumn{2}{|r|}{ Variáveis } \\
\hline \multirow{4}{*}{ 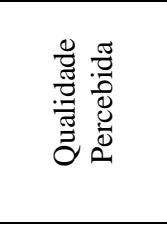 } & QS1 & $\begin{array}{l}\text { Quando nosso principal parceiro logístico promete fazer alguma coisa num determinado prazo, ele } \\
\text { o faz. }\end{array}$ \\
\hline & QS2 & O principal parceiro logístico executa o serviço corretamente na primeira vez. \\
\hline & QS3 & O principal parceiro logístico é confiável na entrega das remessas (é pontual e assertivo, etc). \\
\hline & QS4 & $\begin{array}{l}\text { Temos facilidade para agendar uma coleta de nossas remessas com nosso principal parceiro } \\
\text { logístico. }\end{array}$ \\
\hline 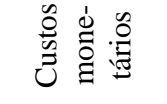 & SC1 & Nosso principal parceiro logístico tem um preço justo. \\
\hline \multirow{3}{*}{ 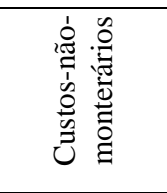 } & $\mathrm{SC} 2$ & $\begin{array}{l}\text { O número de visitas ou reuniões que os nossos colaboradores têm com o principal parceiro } \\
\text { logístico é importante para o bom desenvolvimento do nosso relacionamento. }\end{array}$ \\
\hline & SC3 & O esforço de negociação com o principal parceiro logístico para se chegar a um acordo é aceitável. \\
\hline & $\mathrm{SC} 4$ & $\begin{array}{l}\text { O esforço investido na formação dos nossos funcionários nos produtos e serviços do principal } \\
\text { parceiro logístico é aceitável. }\end{array}$ \\
\hline \multirow{5}{*}{$\begin{array}{l}\tilde{U} \\
0 \\
0 \\
0 \\
0 \\
0 \\
0 \\
0 \\
\tilde{O}\end{array}$} & SC5 & $\begin{array}{l}\text { Custaria muito dinheiro à minha empresa se tivéssemos que trocar nosso principal parceiro } \\
\text { logístico por outro. }\end{array}$ \\
\hline & SC6 & $\begin{array}{l}\text { Demandaria muito tempo à minha empresa se ela tivesse que trocar o principal parceiro logístico } \\
\text { por outro. }\end{array}$ \\
\hline & SC7 & $\begin{array}{l}\text { Demandaria muito esforço à minha empresa se ela tivesse que trocar o principal parceiro logístico } \\
\text { por outro. }\end{array}$ \\
\hline & SC8 & $\begin{array}{l}\text { Se minha empresa trocasse principal parceiro logístico por outro, poderiam aparecer alguns } \\
\text { problemas tecnológicos. }\end{array}$ \\
\hline & SC9 & Nossa empresa se sentiria insegura se tivesse que escolher outro parceiro logístico. \\
\hline \multirow{7}{*}{ 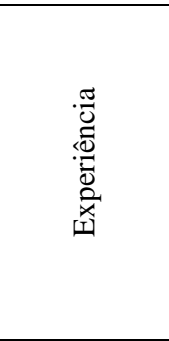 } & EX1 & Os funcionários do principal parceiro logístico são corteses conosco. \\
\hline & EX2 & O relacionamento com os funcionários do principal parceiro logístico é agradável. \\
\hline & EX3 & Os funcionários do principal parceiro logístico compreendem as características do nosso negócio. \\
\hline & EX4 & O principal parceiro logístico possui funcionários que nos dão atenção pessoal. \\
\hline & EX5 & $\begin{array}{l}\text { Quando nossa empresa tem algum problema, o principal parceiro logístico demonstra sincero } \\
\text { interesse em resolvê-lo. }\end{array}$ \\
\hline & EX6 & O comportamento dos funcionários do nosso principal parceiro logístico inspira confiança em nós. \\
\hline & EX7 & $\begin{array}{l}\text { Os funcionários do principal parceiro logístico possuem conhecimento técnico para responder às } \\
\text { nossas perguntas. }\end{array}$ \\
\hline \multirow{4}{*}{ 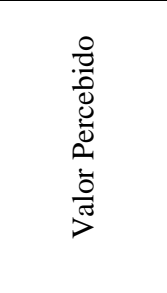 } & VP1 & $\begin{array}{l}\text { Comparando-se com os "sacrifícios" (ex.: preço, investimentos no relacionamento, custos de } \\
\text { coleta), obtemos "benefícios" adequados de nosso principal parceiro. }\end{array}$ \\
\hline & VP2 & $\begin{array}{l}\text { Se comparado aos seus concorrentes, o principal parceiro logístico oferece o melhor valor } \\
\text { ("benefícios"-"sacrifícios"). }\end{array}$ \\
\hline & VP3 & $\begin{array}{l}\text { Se comparado aos seus concorrentes, o principal parceiro logístico exige menos "sacrifícios" pelos } \\
\text { "benefícios" oferecidos. }\end{array}$ \\
\hline & VP4 & $\begin{array}{l}\text { Se comparado aos seus concorrentes, o principal parceiro logístico exige o mesmo nível de } \\
\text { "sacrifícios" mas oferece mais "benefícios". }\end{array}$ \\
\hline \multirow{4}{*}{ 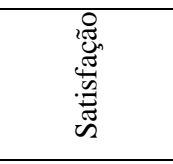 } & ST1 & Nossa empresa está muito satisfeita com o principal parceiro logístico. \\
\hline & ST2 & É um prazer ser cliente do principal parceiro logístico. \\
\hline & ST3 & De forma geral, os serviços do principal parceiro logístico atendem às nossas expectativas. \\
\hline & ST4 & De forma geral, o principal parceiro logístico é uma boa empresa para fazer negócios. \\
\hline \multirow{3}{*}{ 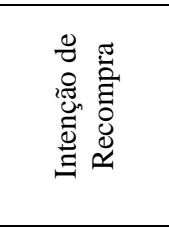 } & IR1 & $\begin{array}{l}\text { Nossa empresa considerará o principal parceiro logístico como a primeira opção em nossa próxima } \\
\text { necessidade de serviços logísticos. }\end{array}$ \\
\hline & IR2 & $\begin{array}{l}\text { Para os próximos seis meses, consideraremos nosso atual parceiro logístico como a primeira opção } \\
\text { dentre os concorrentes. }\end{array}$ \\
\hline & IR3 & $\begin{array}{l}\text { Nós temos a intenção de continuar a ser cliente do nosso principal parceiro logístico nos próximos } \\
\text { anos. }\end{array}$ \\
\hline
\end{tabular}

Quadro 1 - Variáveis da escala proposta Fonte: Elaborado pelos autores.

Inicialmente, as 31variáveis foram traduzidas do inglês para o português, pois todas as escalas originalmente utilizadas foram publicadas na língua inglesa. A tradução reversa foi realizada por tradutor profissional nativo de país de língua inglesa e fluente em língua portuguesa. Os ajustes propostos pelo tradutor foram acatados. Com a finalidade de facilitar a coleta de dados e também conferir maior 
precisão às análises estatísticas posteriores, utilizouse uma escala tipo Likert com 7 pontos, sendo: 1 Discordo totalmente; 2 - Discordo; 3 - Discordo parcialmente; 4 - Indiferente / Não se aplica; 5 Concordo parcialmente; 6 - Concordo; 7 - Concordo totalmente. A seguir, o instrumento completo (31 itens que representaram as variáveis observáveis e questões de identificação do respondente) foi submetido à validação de conteúdo por cinco especialistas. Ajustes semânticos foram incorporados à versão final do questionário.

Antes do início da coleta de dados, realizouse um pré-teste com sete empresários com o perfil de interesse da pesquisa: todos eram de PMEs e usavam serviços logísticos terceirizados. $\mathrm{O}$ instrumento de coleta de dados foi disponibilizado na plataforma SurveyMonkey® e em questionário impresso autoaplicado. A técnica de amostragem utilizada foi a não probabilística por conveniência, delimitada em sua maioria por PMEs e empresários no geral, todos usuários de serviços logísticos de transporte terceirizados, que voluntariamente atenderam ao pedido de participação na pesquisa.

A coleta de dados ocorreu entre 15/09/2015 e 08/11/2015. Foram convidados a participar da pesquisa 2480 empresários, contactados por e-mail fornecido por associações comerciais da região do Triângulo Mineiro e Alto Paranaíba, e também abordados no evento do SEBRAE ( $16^{\mathrm{a}}$ Rodada de Negócios do Brasil Central) na cidade de Uberlândia-MG. Do total de 207 respostas, 182 $(87,92 \%)$ foram coletadas no evento. Após verificação dos dados, foram excluídos 12 respondentes devido à identificação de vieses de respostas estereotipadas, dados perdidos e rasuras.

Os dados foram analisados utilizando Análise Fatorial Exploratória (AFE) e Confirmatória (AFC) e Modelagem de Equações Estruturais com Quadrados Mínimos Parciais (MEEPLS). O tamanho da amostra, para a técnica de MEEPLS, apresentou os requisitos mínimos, com um poder estatístico no modelo de $80 \%$, a um nível de significância de 5\% (Hair Jr, Hult, Ringle \& Sarstedt, 2014).

Os testes de normalidade, por meio da análise de assimetria e curtose (Kline, 2011), linearidade, colinearidade e multicolinearidade (Hair Jr, Black, Babin, Anderson \&Tatham, 2009) foram realizados, e identificadas violações de parâmetros das variáveis SC6_R, SC7_R e EX1 no critério de multicolinearidade. As tratativas foram realizadas e registradas na $\mathrm{AFE}$ e na $\mathrm{AFC}$.

\section{ANÁLISE DOS DADOS}

A maioria dos respondentes encontra-se em Minas Gerais (97\%), especificamente na cidade de
Uberlândia-MG, que representa $78 \%$ do total da amostra, com 152 respondentes. A maioria dos respondentes atua em todo o território nacional (48\%), com predominância na prestação de serviços (43\%). De acordo com o critério da quantidade de funcionários, apenas $10 \%$ da amostra pode ser considerada de grande porte (acima de 250). Já em relação ao critério do faturamento, $17 \%$ das empresas podem ser consideradas de médio ou grande porte. Portanto, cerca de $83 \%$ dos respondentes representam empresas de micro, pequeno e médio portes.

Em relação ao perfil de consumo de serviços logísticos, a maioria dos respondentes $(80 \%)$ consomem até $\mathrm{R} \$ 10 \mathrm{mil} / \mathrm{mês}$, compatível com o porte da maioria das PMEs, e $80 \%$ das remessas enviadas por este negócios possuem valor mercantil, o que denota a importância do serviço logístico de transporte para os elementos da amostra. Vale ressaltar que $80,5 \%$ dos respondentes possuem cargos gerenciais, indicando que as respostas foram fornecidas por pessoas com conhecimento relevante acerca do consumo de serviços logísticos em suas empresas. Verificou-se também que $52 \%$ dos respondentes trabalham predominantemente com um parceiro logístico específico. Ao todo foram citados 54 diferentes parceiros logísticos pelos respondentes, sugerindo um mercado concorrencial neste segmento.

\subsection{Análise Fatorial Exploratória (AFE)}

Executou-se a AFE com as 31 variáveis propostas, e esta foi validada pelo teste de esfericidade de Bartlett e pelo índice de KaiserMeyer-Olkin (KMO). O exame das comunalidades $(>0,50)$ e das cargas cruzadas $(>0,45)$, conforme Hair Jr et al. (2009), apontou para a exclusão de duas variáveis: QS4 e VP1.

A multicolinearidade entre as variáveis SC6_R e SC7_R foi tratada com análises do alfa de Cronbach, da tolerância e do Fator de Inflação da Variância - VIF, levando à exclusão da variável SC6_R.

O modelo foi reespecificado e a AFE novamente validada. A variável SC1 apresentou baixa comunalidade $(0,325)$ e cargas cruzadas, conduzindo à sua exclusão. A AFE foi novamente validada pelo teste de esfericidade de Bartlett e pelo índice KMO de 0,874, atendendo aos critérios estabelecidos na literatura. Os componentes gerados pela AFE foram selecionados pelo critério de autovalores iniciais maiores que 1 , e o poder de explicação do fenômeno manteve-se em $69 \%$ atendendo ao mínimo de 60\% (Hair Jr et al., 2009).

As comunalidades das variáveis ficaram entre 0,496 e 0,824 . Optou-se pela manutenção da variável EX7, única que apresentou comunalidade 
abaixo de 0,50: 0,496, pois a mesma não apresentou cargas cruzadas na matriz de cargas fatoriais rotacionada.

A análise da confiabilidade por meio do Alfa de Cronbach, com um intervalo de confiança de $95 \%$, corroborou a reespecificação, variando entre 0,75 e 0,91 , com a média de 0,87 (muito bom).

\subsection{Análise Fatorial Confirmatória (AFC)}

Para a realização da $\mathrm{AFC}$ (Hair $\mathrm{Jr}$ et al., 2014; Ringle, Silva \& Bido, 2014), criou-se um modelo de mensuração com as variáveis observáveis, e um modelo estrutural com os caminhos sugeridos pela teoria, considerando as 27 variáveis resultantes da AFE (Figura 1). O algoritmo para o PLS utilizado foi o sistema de ponderação de caminhos, com peso inicial igual a 1 e 300 iterações. Para a realização dos cálculos, eliminou-se 4 respondentes com dados parcialmente perdidos da amostra de 195, restando para a AFC e para a MEE uma amostra de 191 respondentes.

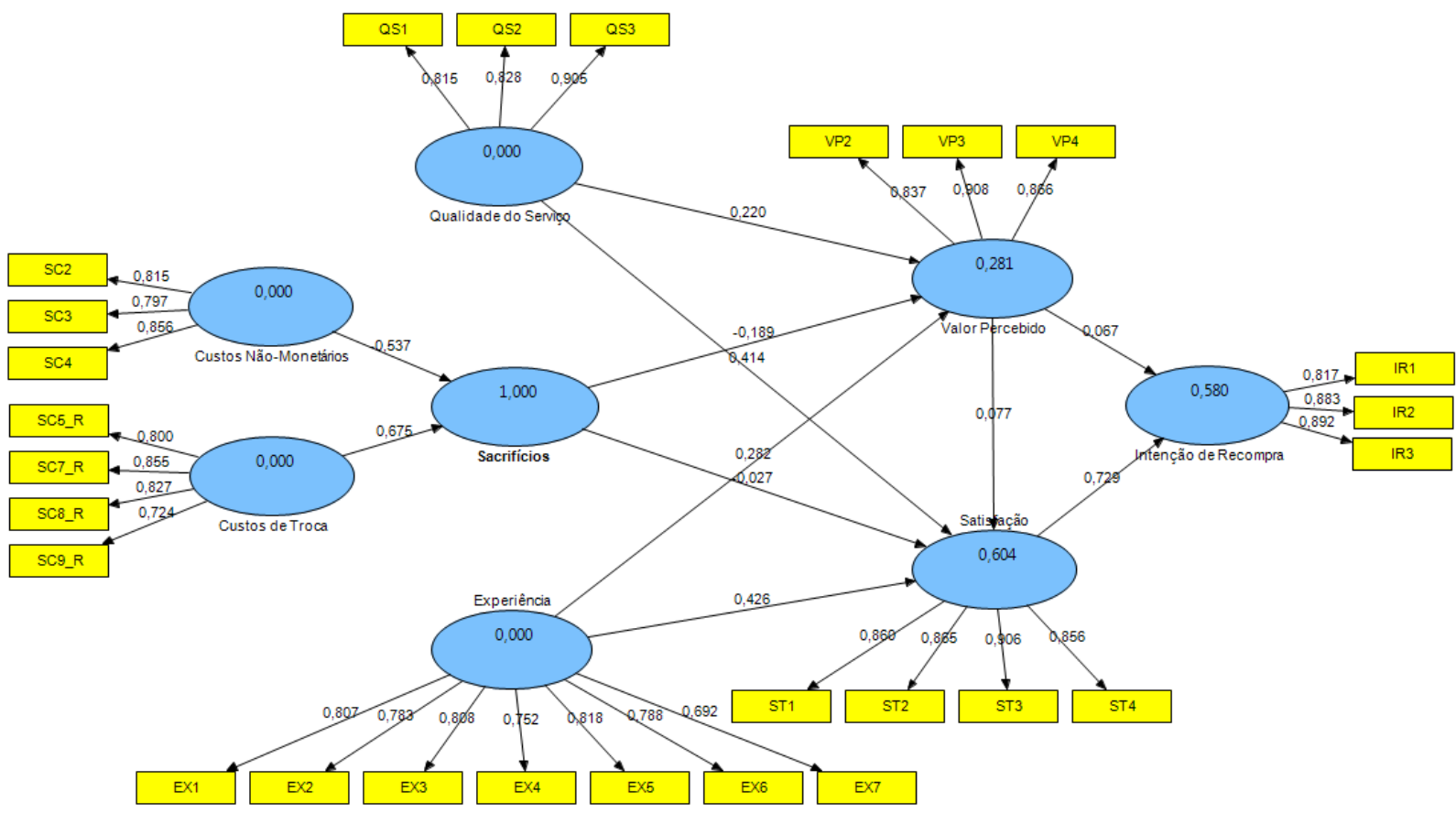

Figura 1 - Modelo de mensuração e estrutural inicial Fonte: Elaborado pelos autores.

Para avaliar as validades convergente e discriminante no modelo de mensuração (Hair $\mathrm{Jr}$ et al., 2014) verificou-se as cargas fatoriais das variáveis, e o item EX7 do modelo obteve carga abaixo de 0,70 (0,692). Os demais valores oscilaram entre 0,72 e 0,90. Já para a análise da AVE (comunalidade de um constructo - parâmetro: $>0,50)$, verificou-se que o constructo sacrifícios violou o parâmetro $(0,44)$. Os demais constructos oscilaram entre 0,60 e 0,75 .

Ainda na validade convergente (Hair $\mathrm{Jr}$ et al., 2014), observou-se na confiabilidade composta (CC - parâmetro: >0,70), que houve violação de parâmetro no constructo sacrifícios $(0,16)$. Os demais constructos tiveram CC entre 0,86 e 0,92 . O constructo sacrifícios foi reavaliado, excluindo-se variáveis SC3 e SC4, e mantendo-se apenas a variável SC2. Após o ajuste, obteve-se:
- Cargas fatoriais entre 0,72 e 0,90 , à exceção da variável observável EX7, que obteve carga levemente abaixo de $0,70(0,69)$, mantida no modelo;

- AVEs entre 0,54 e 0,75;

- CC entre 0,75 e 0,92;

- Critério de Fornell e Lacker atendido; e

- Critério de cargas cruzadas atendido.

\subsection{Modelagem de Equações Estruturais com Partial Least Squares - PLS (MEEPLS)}

Atendidos os parâmetros de validade discriminante e convergente, apresenta-se a avaliação do modelo estrutural e a MEEPLS, considerando 4 variáveis exógenas: qualidade do serviço (3 variáveis), sacrifícios, constructo de 
segunda ordem composto por duas variáveis exógenas: custos não-monetários (1 variável) e custos de troca (4 variáveis), experiência (7 variáveis); e três variáveis endógenas: valor percebido (3 variáveis), satisfação (4 variáveis) e intenção de recompra (3 variáveis). O Coeficiente de Determinação $\left(\mathrm{R}^{2}\right)$ ajustado, Coeficiente de Redundância Cross-validado $\left(\mathrm{Q}^{2}\right)$, o Tamanho do Efeito $\left(\mathrm{f}^{2}\right)$ e o Coeficiente de Caminhos (p) foram analisados conforme método proposto por Hair $\mathrm{Jr}$ et al. (2014). O coeficiente de caminhos (p) foi comparado ao t-value, para um intervalo de confiança de $95 \%$. Os t-values foram gerados pela técnica de Bootstrapping do SMARTPLS 2.0 M3 (Ringle, Wende \& Will, 2005), e considerou-se para a análise, os efeitos totais (Ringle et al., 2014).

$\mathrm{O}$ modelo estrutural proposto apresentou algumas relações entre os constructos pouco significantes. Com a finalidade de sugerir um modelo mais adequado, procedeu-se com a reespecificação do modelo, conforme Ringle et al. (2014), baseando-se na significância (t-value) das relações entre os constructos, bem como na teoria que sustenta este trabalho (Quadro 2).

\begin{tabular}{|c|c|c|}
\hline Reespecificação & Modificação & $\begin{array}{c}\text { Resultados não significantes } \\
\text { Parâmetro: } t>1,96\end{array}$ \\
\hline \multirow{2}{*}{1} & $\begin{array}{c}\text { Exclusão da variável SC2. O constructo } \\
\text { Sacrifícios tornou-se apenas Custos de } \\
\text { Troca (CT) }\end{array}$ & $\mathrm{CT} \rightarrow \mathrm{ST}=0,175142$ \\
& $\mathrm{VP} \rightarrow \mathrm{IR}=0,152161$ \\
& $\mathrm{VP} \rightarrow \mathrm{IRT}=1,459195$ \\
& Exclusão da relação $\mathrm{CT} \rightarrow \mathrm{ST}=0,175142$ & $\mathrm{CT} \rightarrow \mathrm{ST}=0,956532$ \\
& $\mathrm{CT} \rightarrow \mathrm{IR}=1,180401$ \\
& $\mathrm{VP} \rightarrow \mathrm{IR}=1,449350$ \\
\hline 3 & Exclusão da relação $\mathrm{VP} \rightarrow \mathrm{ST}=1,152464$ & $\mathrm{VP} \rightarrow \mathrm{ST}=1,152464$ \\
\hline 4 & Exclusão da relação $\mathrm{VP} \rightarrow \mathrm{IR}=1,185700$ & $\mathrm{CT} \rightarrow \mathrm{IR}=0,950366$ \\
& & $\mathrm{VP} \rightarrow \mathrm{IR}=1,185700$ \\
\hline
\end{tabular}

Quadro 2 - Reespecificações do modelo estrutural Fonte: Elaborado pelos autores.

Apesar de a $4^{\mathrm{a}}$ reespecificação apresentar resultados significantes para todas as relações propostas, o constructo VP não se relacionou com os constructos ST e IR, apresentando pouca aderência à teoria e pesquisas sobre a temática. Por este motivo, e baseando-se na proposição teórica de Fishbein e Azjen (1975), uma $5^{\text {a }}$ reespecificação foi proposta, ordenando os constructos exógenos (qualidade do serviço, custos de troca e experiência) como antecedentes do valor percebido (cognição), que por sua vez influencia a satisfação (atitude), e esta influenciando a intenção de recompra (intenção que gera comportamento).

Para validar modelo o novo modelo estrutural, realizou-se novamente os testes de $R^{2}$ ajustado, $\mathrm{Q}^{2}, \mathrm{f}^{2}$ e o Coeficiente de Caminhos (p), conforme método proposto por Hair Jr et al. (2014) e Ringle et al. (2014). O coeficiente de caminhos foi comparado com o t-value, para um intervalo de confiança de $95 \%$ e verificou-se que os custos de troca apresentaram relações não-significantes.

Os resultados das comparações de $\mathrm{R}^{2}, \mathrm{Q}^{2}$, $\mathrm{f}^{2}, \mathrm{p}$ e t-value apontaram para uma possível melhora preditiva do modelo caso o constructo custos de troca fosse excluído. No entanto, antes de proceder com a exclusão, avaliou-se indicadores de coeficiente de caminhos (p) com efeitos totais e tvalue do modelo reespecificado para dois grupos distintos: respondentes que apontaram trabalhar com apenas 1 parceiro logístico $(n=100)$ e aqueles que têm mais de um parceiro logístico $(n=91)$. O resultado apresentou t-values e coeficientes de caminhos (p) sem significância nas duas amostras. Somando-se este resultado com as análises dos indicadores de predição do modelo $\left(\mathrm{R}^{2}, \mathrm{Q}^{2}\right.$ e $\left.\mathrm{f}^{2}\right)$, optou-se por um modelo mais parcimonioso que excluiu o constructo custos de troca. Assim, a Figura 2 representa o modelo reespecificado final: 


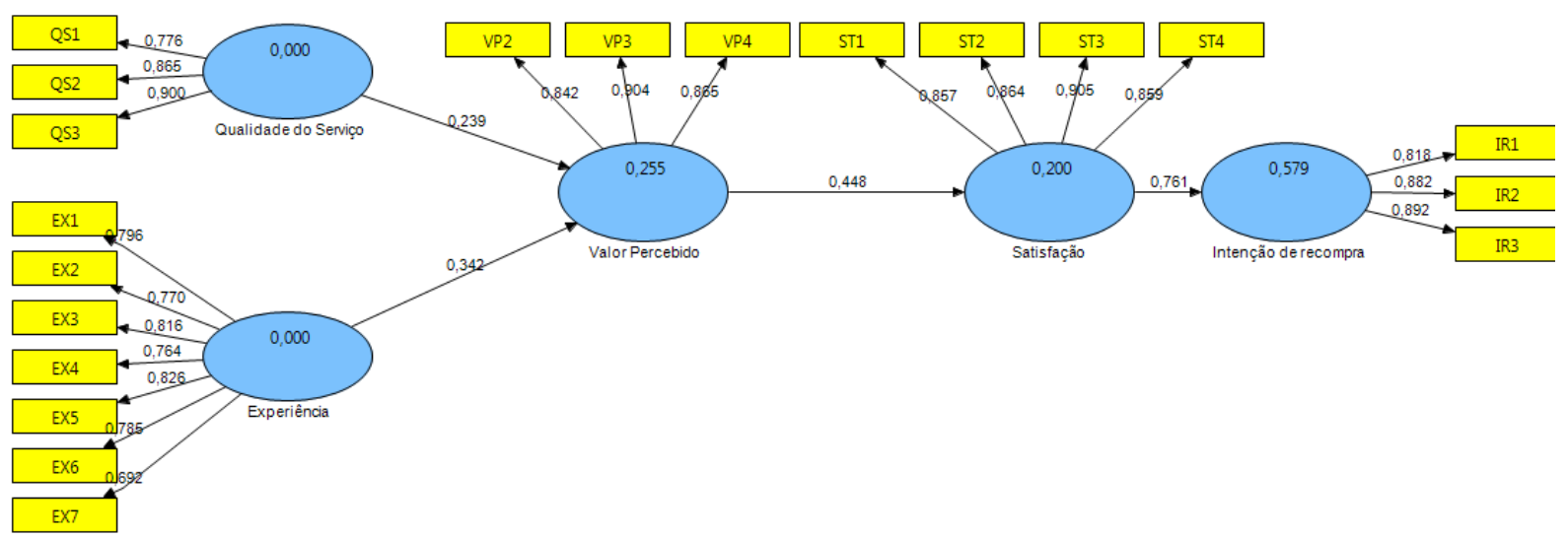

Figura 2 - Modelo de mensuração e estrutural reespecificado final Fonte: Elaborado pelos autores. a 3).

Os parâmetros de avaliação do modelo estrutural reespecificado foram novamente calculados (Tabelas 1

Tabela 1 - $\mathrm{R}^{2}$ Ajustado e $\mathrm{Q}^{2}$

\begin{tabular}{|c|c|c|c|c|}
\hline \multirow{2}{*}{ Constructo } & $\mathbf{R}^{2}$ Ajustado & Resultado & $\mathbf{Q}^{2}$ & Resultado \\
\hline & \multicolumn{2}{|c|}{$\begin{array}{c}\text { Parâmetros: 2\%-efeito pequeno; } 13 \% \text {-efeito médio; } \\
26 \% \text {-efeito grande }\end{array}$} & \multicolumn{2}{|c|}{ Parâmetro: $\mathrm{Q}^{2}>0$} \\
\hline IR & 0,590619 & Efeito Grande & 0,4248 & Adequado \\
\hline ST & 0,204548 & Efeito Médio & 0,1529 & Adequado \\
\hline VP & 0,260144 & Efeito Grande & 0,1936 & Adequado \\
\hline
\end{tabular}

Fonte: Elaborado pelos autores.

Tabela 2 - $\mathrm{f}^{2}$

\begin{tabular}{|c|c|c|c|c|}
\hline \multirow{3}{*}{$\begin{array}{c}\text { Constructo } \\
\text { IR }\end{array}$} & \multicolumn{4}{|c|}{$\begin{array}{l}\text { Constructo Excluído para Análise do Efeito }\left(\mathbf{f}^{\mathbf{2}}\right) \\
\text { Parâmetro: 0,02-pequeno; 0,15-médio; 0,35-grande }\end{array}$} \\
\hline & \multicolumn{2}{|c|}{ Experiência } & \multicolumn{2}{|c|}{ Qualidade do Serviço } \\
\hline & 0,000 & Pequeno & 0,000 & Pequeno \\
\hline ST & 0,001 & Pequeno & 0,000 & Pequeno \\
\hline VP & 0,117 & Médio & 0,056 & Médio \\
\hline
\end{tabular}

Fonte: Elaborado pelos autores.

Tabela 3 - Efeitos Totais, Coeficientes de Caminhos (p) e t-value

\begin{tabular}{|c|c|c|c|}
\hline \multirow{2}{*}{ Constructo } & Caminhos & $p$ & t-value \\
\hline \multirow{3}{*}{ EX } & $\mathrm{EX} \rightarrow \mathrm{IR}$ & 0,116499 & $2,876347^{*}$ \\
\cline { 2 - 4 } & $\mathrm{EX} \rightarrow \mathrm{ST}$ & 0,153144 & $3,040161^{*}$ \\
\cline { 2 - 4 } & $\mathrm{EX} \rightarrow \mathrm{VP}$ & 0,342086 & $4,36313^{*}$ \\
\hline \multirow{3}{*}{$\mathrm{QS}$} & $\mathrm{QS} \rightarrow \mathrm{IR}$ & 0,081331 & $2,171864^{*}$ \\
\cline { 2 - 4 } & $\mathrm{QS} \rightarrow \mathrm{ST}$ & 0,106914 & $2,179295^{*}$ \\
\cline { 2 - 4 } & $\mathrm{QS} \rightarrow \mathrm{VP}$ & 0,238819 & $2,692441^{*}$ \\
\hline \multirow{2}{*}{$\mathrm{ST}$} & $\mathrm{ST} \rightarrow \mathrm{IR}$ & 0,760715 & $19,892403^{*}$ \\
\hline \multirow{2}{*}{$\mathrm{VP}$} & $\mathrm{VP} \rightarrow \mathrm{IR}$ & 0,340556 & $5,414938^{*}$ \\
\cline { 2 - 4 } & $\mathrm{VP} \rightarrow \mathrm{ST}$ & 0,447679 & $5,902841^{*}$ \\
\hline
\end{tabular}

Legenda: *Significante-t-value>1,96 / ** Não Significante Fonte: Elaborado pelos autores. 
Observa-se que, com a reespecificação final, os indicadores de $\mathrm{R}^{2}$ Ajustado e $\mathrm{Q}^{2}$ continuaram a apresentar resultados semelhantes para a acurácia do constructo intenção de recompra. Portanto, tal reespecificação apresentou um modelo mais parcimonioso sem abrir mão da acurácia preditiva.

\section{DISCUSSÃO DOS RESULTADOS}

O modelo final apresentou mais parcimônia sem perder acurácia preditiva da intenção de recompra. A partir deste modelo, e com base na Tabela 3, verificou-se que as Hipóteses H3 e H4, referentes às relações com o constructo Sacrifícios foram rejeitadas, e as demais não-rejeitadas.

No que se refere às hipóteses ligadas à qualidade do serviço (H1 e H2), as variáveis observáveis propostas apresentaram boa aderência ao modelo proposto, e na amostra estudada o constructo apresentou relações significantes tanto com a satisfação quanto com o valor percebido. A análise dos efeitos totais dos coeficientes de caminho (Tabela 3) mostrou que a qualidade do serviço também influencia positivamente a intenção de recompra. O modelo final apresentou melhor relação da qualidade do serviço com o valor percebido, em detrimento da relação com a satisfação e a intenção de recompra. Os dados da Tabela 3 mostram que, no caso da amostra pesquisada, apesar de influenciar positivamente a satisfação e a intenção de recompra, a qualidade do serviço adequa-se melhor quando forma diretamente o valor percebido.

Quanto às hipóteses ligadas aos sacrifícios rejeitadas (H3 e $\mathrm{H} 4)$, é importante ressaltar que diversas inconsistências foram encontradas $\mathrm{e}$ relatadas durante os testes metodológicos. Tais inconsistências culminaram na exclusão do constructo sacrifícios no contexto do modelo proposto.

Verificou-se na AFE que a exclusão da variável custo monetário (SC1) não sugere necessariamente que os gestores entrevistados não avaliam custos monetários. A avaliação dos sacrifícios como um todo pode ser também encontrada nas variáveis do constructo valor percebido (VP2-VP4). A exclusão desta variável sugere que, para a amostra pesquisada, custos monetários não apresentaram relações significantes com o restante do modelo proposto para predizer valor percebido, satisfação ou intenção de recompra.

No que se refere aos custos de troca (SC5SC9), estes foram retirados apenas na última reespecificação. Antes de sua exclusão, verificou-se se os custos de troca apresentavam significância entre o grupo de respondentes que apontaram apenas um parceiro logístico, e o grupo que apontou mais de um parceiro logístico. Os resultados apontaram que os dois grupos se comportaram da mesma maneira, apresentando relações não-significantes. Portanto, sugere-se que, para o grupo de respondentes que compuseram a amostra, os custos de troca não influenciam de forma significativa a formação do valor percebido, da satisfação e da intenção de recompra, o que pode indicar que é fácil mudar de fornecedor (Burnham et al., 2003) de forma segura e viável (Yanamandram \& White, 2006).

O cenário de níveis não-significantes relacionados entre custos de troca, satisfação e intenção de recompra é melhor explicado se vier à tona o trabalho de Patterson (2004), o qual sugere que, quando barreiras de troca são percebidas como baixas, consumidores estão livres para trocarem de fornecedor caso estejam insatisfeitos, ou para manterem uma relação com o atual fornecedor caso estejam satisfeitos. Assim, é possível que a percepção do valor seja assimetricamente influenciada pela relevância do desempenho dos serviços mensurados na qualidade e na experiência, e pouco influenciada pelos sacrifícios que os respondentes empenham para obter benefícios.

O terceiro constructo exógeno mantido no modelo final foi a experiência, ligada às hipóteses H5 e H6. Este constructo apresentou resultados muito homogêneos durante a aplicação de todas as técnicas estatísticas e todas as variáveis observáveis foram mantidas no modelo reespecificado final, apresentando níveis de significância relevantes. A experiência apresentou níveis de significância, coeficientes de caminho e efeitos totais (Tabela 3) maiores que a qualidade do serviço. Além disto, o constructo apresentou maior efeito $\left(\mathrm{f}^{2}\right)$ no modelo proposto (Tabela 2), ratificando sua importância preponderante em relação à qualidade do serviço na predição dos constructos endógenos. Tais resultados corroboram a importância dos aspectos mensurados no constructo experiência para a formação do valor percebido, da satisfação e da intenção de recompra, mostrando que os consumidores B2B de serviços logísticos de transporte, em especial as PMEs, articulam suas vivências em encontros de uso e serviços para julgar a excelência ou a superioridade de suas experiências (Lemke et al., 2011).

Os resultados das hipóteses $\mathrm{H} 5$ e $\mathrm{H} 6$ alinharam-se aos achados de Irene et al. (2008): por meio da experiência vivenciada na prestação dos serviços, o consumidor B2B forma suas percepções de valor e os encontros de serviços podem ser considerados como um importante antecedente da avaliação que os consumidores fazem da performance de serviços. No entanto, é necessário considerar a falta de tempo e recursos que os PMEs vivenciam no dia-a-dia, e é de se esperar que encontrem dificuldade na avaliação de parceiros logísticos, sendo comum que tal lacuna de 
mensuração objetiva de desempenho seja suprida com o desenvolvimento de relacionamentos cooperativos e/ou baseados na personalidade do dono da PME, ou mesmo priorizando fornecedores mais estáveis que podem minimizar perdas (Pressey et al., 2009). Esta pode ser a realidade vivenciada pelas PMEs respondentes. Assim, é possível sugerir que aspectos ligados à experiência das PMEs sejam preponderantes na formação do valor percebido. Se o parceiro logístico entende as necessidades do seu cliente, é flexível, cortês, agradável, atende de forma pessoal, inspira confiança, resolve os problemas quando estes acontecem e possui conhecimento técnico, muitos problemas enfrentados pelas PMEs no desafio de gerenciar sua cadeia de suprimentos estarão resolvidos. Experiências positivas vividas na lida com o parceiro logístico apresentam potencial de se relacionarem de forma positiva com a percepção de valor, a satisfação e a intenção de recompra.

Ainda na discussão acerca da experiência, verificou-se que houve forte influência deste contructo no valor percebido (Tabela 3), o que corrobora as proposições de Flint et al. (1997), os quais sugerem que o valor percebido depende da experiência para se concretizar. Os valores de julgamento, no caso dos serviços logísticos, parecem ser preponderantes, como também propõem Ravald e Grönroos (1996). Estudos como o de Fiol et al. (2011) encontraram alta correlação entre aspectos experienciais e o valor percebido no ambiente B2B.

No que se refere às hipóteses $\mathrm{H} 7$ e H9, ficou evidente a baixa influência do valor percebido tanto na satisfação como na intenção de recompra no modelo inicialmente proposto. Foram necessárias reespecificações até que o constructo valor percebido pudesse relacionar-se de forma relevante com os demais, operando melhor como um antecedente da satisfação, e não influenciando diretamente a intenção de recompra. A análise dos efeitos totais (Tabela 3) não rejeita, portanto, as hipóteses H7 e H9: o valor percebido influencia diretamente a satisfação e indiretamente a intenção de recompra.

Esta posição do valor percebido como um antecedente corrobora modelos como o de Eggert e Ulaga (2002), que o consideram como um constructo eminentemente cognitivo, o qual influencia a satisfação, que por sua vez influencia a intenção de recompra. Assim, a presente pesquisa alinhou-se ao proposto por Chaudhuri (2006), que indica a satisfação como um melhor preditor de intenções comportamentais do que o valor percebido.

A satisfação foi analisada na presente pesquisa por uma ótica predominantemente afetiva e emocional, alinhada à proposta de Eggert e Ulaga (2002). Ficou evidenciado nos resultados que aspectos emocionais são preponderantes na predição da intenção de recompra para a amostra pesquisada: clientes satisfeitos possuem mais propensão à compra. A estrutura cognição-afeto-conação-ação (Fishbein \& Ajzen, 1975) pôde ser verificada no presente estudo: a satisfação foi formada por expectativas que geraram atitudes, que por sua vez geram intenções comportamentais.

$\mathrm{Na}$ discussão acerca da relação da qualidade e da experiência com a satisfação, conforme encontrado em Oliver (1994), a presente pesquisa verificou que tanto variáveis cognitivas quanto afetivas contribuem de maneira significativa na construção da satisfação, pois aparentemente, dois mecanismos operaram em conjunto nas mentes dos respondentes: um envolveu a avaliação comparativa de resultados funcionais no constructo valor percebido ("o que o serviço me deu”), e outro relacionado a como o serviço influenciou o afeto, mensurado no constructo satisfação, provavelmente mais influenciado pela experiência.

A conotação emocional do constructo satisfação corroborada pelos respondentes sugere que, apesar de afetos não dependerem de cognições racionais, todas as cognições racionais são acompanhadas de afeto (Chaudhuri, 2006), e os resultados encontrados na presente pesquisa sugerem que aspectos emocionais mesclam-se à racionalidade dos gestores de PMEs pesquisados.

O comportamento do constructo satisfação no modelo final proposto evidenciou que o fator atitudinal pós-consumo da intenção de recompra, para a presente pesquisa, é fortemente formado pela satisfação (Fishbein \& Azjen, 1975), que não rejeitou a hipótese $\mathrm{H} 8$, e concorda com estudos que verificaram tal relação, como os de Kumar (2002) e Patterson (2004). O mesmo resultado foi encontrado também por Eggert e Ulaga (2002), sustentando que a proposição do modelo final possui aderência com outros estudos empíricos e proposições teóricas.

\section{CONSIDERAÇÕES FINAIS}

O modelo reespecificado final apresenta diversas convergências com proposições teóricas apresentadas neste estudo. Uma questão prioritária apresentada por Lindgreen et al. (2012) "como valor, qualidade e satisfação dos consumidores estão conceitualmente e empiricamente relacionados?" encontrou evidências de que, na presente amostra, valor percebido atua como um antecedente da satisfação, e esta, por sua vez, apresenta-se como melhor preditora de intenção de recompra.

Boksberger e Melsen (2011) sugeriram investigações aprofundadas em atributos formadores do valor percebido e suas consequências nas intenções de compra e os resultados deste estudo indicaram que, apesar de importantes, os sacrifícios 
apresentaram-se sem significância estatística no relacionamento entre clientes PMEs e fornecedores de serviços logísticos de transporte. Estes clientes valorizam em maior medida aspectos ligados à qualidade do serviço e à experiência vivenciada na prestação do serviço.

Outro importante achado refere-se à influência dos sacrifícios, em especial os custos de troca na formação do valor percebido, da satisfação e da intenção de recompra. A aparente facilidade de mudar de transportador parece não influenciar a formação do valor. Visto sob a perspectiva do modelo proposto como um todo, tal achado pode indicar que, para a amostra pesquisada, a força da manutenção do relacionamento e da consequente repetição da compra não está na dificuldade de se desvincular deste relacionamento e trocar de parceiro logístico, mas sim das possibilidades de ganhos que as PMEs obtêm quando mantêm relacionamentos baseados em qualidade e experiência em níveis aceitáveis.

Desta forma, os achados da presente pesquisa verificaram que, de fato, a formação do valor percebido, da satisfação e da intenção de recompra apresentam-se como dimensões complexas e de alta abstração (Zeithaml, 1988) podendo ser influenciados por dimensões racionais e não-racionais (Mencarelli \& Rivière, 2015). Esta constatação vai ao encontro do que Pressey et al. (2009) sugeriram: apesar de avaliarem de forma deficiente e pouco objetiva seus parceiros logísticos, é comum que tal lacuna de mensuração objetiva de desempenho seja suprida com o desenvolvimento de relacionamentos cooperativos e/ou baseados na personalidade do dono do PME, ou mesmo priorizando fornecedores mais estáveis que podem minimizar perdas. Por isto, é provável que as PMEs, ao elegerem como formadores do valor percebido a experiência e qualidade, e por serem fortemente influenciadas pela satisfação para intentarem recompras, estejam evidenciando um comportamento peculiar que permita gerenciar sua cadeia de suprimentos por meio do estabelecimento de relacionamentos pessoais e envoltos em mais subjetividades do que racionalmente se espera de um empresário.

No âmbito teórico, pôde-se propor um modelo que prediz com acurácia razoável a intenção de recompra de PMEs consumidoras de serviços logísticos, tendo como preditor dela a satisfação, e desta, o valor percebido.

Por ser uma fonte de vantagem competitiva, o valor percebido precisa ser adequadamente gerenciado pelas empresas (Anderson \& Narus, 1999). Ao apresentar um modelo preditivo onde o valor percebido desempenha um papel de antecedente da satisfação, a presente pesquisa municia o ambiente gerencial com informações que podem subsidiar análises de mercado e segmentação de produtos logísticos, posicionamento de produtos e marcas ou mesmo planejamento e desenvolvimento de produtos e marcas ligados a este segmento.

Por se tratar de uma pesquisa que utilizou uma amostra por conveniência, os resultados da presente investigação não puderam ser extrapolados para outros ambientes, e nem possuem validade nomológica. Em futuras investigações, sugere-se que a mesma pesquisa seja realizada em outros contextos mercadológicos do país. Os achados do presente estudo, por terem sido analisados utilizando-se uma amostra por conveniência, apresentam validade interna e não podem ser extrapolados para o universo que representa o mercado de serviços logísticos de transporte como um todo.

\section{REFERÊNCIAS}

Anderson, J. C. \& Narus, J. A. (1999). Business Market Management: Understanding, Creating, and Delivering Value. New Jersey: Prentice-Hall.

Bagozzi, R. P., Gürhan-Canli, Z. \& Priester, J. R. (2002). The social psychology of consumer behavior. Buckingham: Open University Press.

Bitner, M. J., Booms, B. H., \& Tetreault, M. S. (1990). The service encounter: diagnosing favorable and unfavorable incidents. The Journal of Marketing, 54, pp.71-84.

Blocker, C. P. (2011). Modeling customer value perceptions in cross-cultural business markets. Journal of Business Research, 64(5), pp.533-540.

Boksberger, P. E., \& Melsen, L. (2011). Perceived value: a critical examination of definitions, concepts and measures for the service industry. Journal of Services Marketing, 25(3), pp. 229-240.

Burnham, T. A., Frels, J. K., \& Mahajan, V. (2003). Consumer switching costs: a typology, antecedents, and consequences. Journal of the Academy of marketing Science, 31(2), 109-126.

Chaudhuri, A (2006). Emotion and reason in consumer behavior. Oxford: Elsevier.

Confederação Nacional Dos Transportes (2014).

Plano CNT de transporte e logística 2014.

Disponível em:

<http://www.cnt.org.br/Paginas/Plano-CNT-deLog\%C3\%ADstica.aspx >. Acesso em: 25 set.2014. 
Cronin, J. J., Brady, M. K., \& Hult, G. T. M. (2000). Assessing the effects of quality, value, and customer satisfaction on consumer behavioral intentions in service environments. Journal of retailing, 76(2), pp. 193-218.

Drumond, M., \& Toaldo, A. M. M. (2009). Implementação de estratégias de marketing, competitividade e fornecimento de valor em PMEs. Anais do Encontro Nacional da Associação Nacional de Pós-Graduação e Pesquisa em Administração. São Paulo, SP, Brasil, 36.

Eggert, A., \& Ulaga, W. (2002). Customer perceived value: a substitute for satisfaction in business markets?. Journal of Business \& Industrial Marketing, 17(2/3), pp. 107-118.

Figueiredo, K. F., \& Mora, D. M. M. (2009). A Segmentação dos Operadores Logísticos no Mercado Brasileiro de acordo com suas Capacitações para Oferecer Serviços. $R A C$ Eletrônica, 3(1), pp. 123-141.

Fiol, L. J. C., Tena, M. A. M., \& García, J. S. (2011). Multidimensional perspective of perceived value in industrial clusters. Journal of Business \& Industrial Marketing, 26(2), pp. 132-145.

Fishbein, M.; Ajzen, I. (1975). Belief, attitude, intention and behavior: an introduction to theory and research. Reading, MA: Addison-Wesley Publising Company.

Flint, D. J., Woodruff, R. B., \& Gardial, S. F. (1997). Customer value change in industrial marketing relationships: a call for new strategies and research. Industrial Marketing Management, 26(2), pp. 163-175.

Fornell, C., Johnson, M. D., Anderson, E. W., Cha, J., \& Bryant, B. E. (1996). The American customer satisfaction index: nature, purpose, and findings. The Journal of Marketing, pp.7-18.

Gil, A. C. (2002). Como elaborar projetos de pesquisa. $4^{\mathrm{a}}$ ed. São Paulo: Atlas.

Hair, J. F., Black, W. C., Babin, B. J., Anderson, R. E., \&Tatham, R. L. (2009). Análise multivariada de dados. Porto Alegre: Bookman.

Hair Jr, J. F., Hult, G. T,. M., Ringle C. M. \& Sarstedt, M. (2014). A primer on partial least squares structural equation modeling (PLS-SEM). Thousand Oaks: SAGE Publications.

Hong, P. \& Jeong, J. (2006). Supply chain management practices of SMEs: from a business growth perspective. Journal of Enterprise Information Management, 19 (3), pp. 292-302. Irene, G., Berenguer G., Cervera, A. (2008). The roles of service encounters, service value, and job satisfaction in achieving customer satisfacion in business relationships. Industruial Marketing Management, (37), pp. 921-939.

Johnston, R., \& Kong, X. (2011). The customer experience: a road-map for improvement. Managing Service Quality: An International Journal, 21(1), pp. 5-24.

Kline, R. B. (2011). Principles and practice of structural equation modeling. $3^{\text {rd }}$ ed. New York: The Guilford Press.

Kumar, P. (2002). The impact of performance, cost, and competitive considerations on the relationship between satisfaction and repurchase intent in business markets. Journal of Service Research, 5(1), pp. 55-68.

Lacerda, T. S., \& Mendonça, B. Q. (2010). Marketing B2B: mapeamento dos trabalhos acadêmicos no Brasil de 1998 a 2007. Revista de Administração da UFSM, 3(2), pp. 219-229.

Lakatos, E. M.; Marconi, M. D. A. (2000). Metodologia Científica. $3^{\mathrm{a}}$ ed. São Paulo: Atlas.

Lam, S. Y., Shankar, V., Erramilli, M. K., \&Murthy, B. (2004). Customer value, satisfaction, loyalty, and switching costs: an illustration from a business-to-business service context. Journal of the Academy of Marketing Science, 32(3), pp. 293-311.

Lapierre, J. (2000). Customer-perceived value in industrial contexts. Journal of Business \& Industrial Marketing, 15(2/3), pp. 122-145.

Lemke, F., Clark, M., \& Wilson, H. (2011). Customer experience quality: an exploration in business and consumer contexts using repertory grid technique.Journal of the Academy of Marketing Science, 39(6), pp. 846-869.

Lindgreen, A., Hingley, M. K., Grant, D. B., \& Morgan, R. E. (2012). Value in business and industrial marketing: Past, present, and future. Industrial Marketing Management, 41(1), pp. 207-214.

McDougall, G. H., \& Levesque, T. (2000). Customer satisfaction with services: putting perceived value into the equation. Journal of services marketing, 14(5), pp. 392-410.

Mello, S. C. B. D., \& Leão, A. L. M. D. S. (2008). Uma Revisão Crítica Uma Revisão Crítica sobre o 
Uso de Diferentes Conceitos de Valor no Marketing. RAC-Eletrônica, 2 (1), pp. 37-53.

Mencarelli, R., \& Rivière, A. (2015). Perceived value in B2B and B2C: A comparative approach and cross-fertilization. Marketing Theory, 15(2), pp. 201-220.

Molinari, L. K., Abratt, R., \& Dion, P. (2008). Satisfaction, quality and value and effects on repurchase and positive word-of-mouth behavioral intentions in a B2B services context. Journal of Services Marketing, 22(5), pp. 363-373.

Oliver, R. L. (1994). Conceptual issues in the structural analysis of consumption emotion, satisfaction, and quality: evidence in a service setting. In Allen, C. \&; John, D. R. (Ed). NAAdvances in Consumer Research, 21.

Oliver, R. L. (1996). Satisfaction: a behavioral perspective on the consumer. Singapore: Mc-GrawHill.

Oliver, R. L. (2006). Customer satisfaction research. In: Grover, R.\& Vriens, M. (Ed). The handbook of marketing research: Uses, misuses, and future advances. Thousand Oaks, California: Sage Publications.

Ozmen, E. S., Oner, M. A., Khosrowshahi, F., \& Underwood, J. (2013). SME buying behaviour: Literature review and an application agenda. The Marketing Review, 13(2), pp. 207-227.

Parasuraman, A., Zeithaml, V. A. \& Berry, L. L.,(1988). SERVQUAL: a multiple-item scale for measuring consumer perceptions os service quality. Journal of Retailing, 64(1), pp.12-40.

Patterson, P. G.(2004). A contingency model of behavioral intentions in a services context. European Journal of Marketing, 8(9/10), pp.13041315.

Pressey, A. D., Winklhofer, H. M. \& Tzokas, N. X.(2009). Purchasing practices in small-to mediumsized enterprises:An examination of strategic purchasing adoption, supplier evaluation and supplier capabilities. Journal of Purchasing \& Supply Management, 15, pp.214-226.

Ravald, A., \& Grönroos, C. (1996). The value concept and relationship marketing. European journal of marketing, 30(2), pp.19-30.

Ringle, C. M., Silva, D. \& Bido, D. D. S. (2014). Modelagem de equações estruturais com utilização do SmartPLS. REMark, 13(2), pp. 56-73.
Rocha, A. D. \& Silva, J. F. D. (2006). Marketing de serviços: retrospectiva e tendências. Rev. $a d m$. empresas.[online], 46(4), pp.1-9.

Serviço de Apoio às Micro e Pequenas Empresas (2013). Os donos de negócio no Brasil, por regiões e por unidades da Federação. Série de Estudos e Pesquisas. Disponível em:

<http://gestaoportal.sebrae.com.br/customizado/est udos-e-pesquisas/temas-estrategicos/perfil-dospequenos-negocios/apresentacao > . Acesso em: 04 jul. 2014.

Ringle, C. M., Wende, S., \& Will, A. (2005). SmartPLS 2.0.M3. Hamburg: SmartPLS, http://www.smartpls.de.

Spiteri, J. M., \& Dion, P. A. (2004). Customer value, overall satisfaction, end-user loyalty, and market performance in detail intensive industries. Industrial Marketing Management, 33(8), pp. 675-687.

Ulaga, W., \& Chacour, S. (2001). Measuring customer-perceived value in business markets: a prerequisite for marketing strategy development and implementation. Industrial Marketing Management, 30(6), pp. 525-540.

Varadarajan, R. (2010). Strategic marketing and marketing strategy: domain, definition, fundamental issues and foundational premises. Journal of the Academy of Marketing Science, 38(2), pp.119-140.

Yanamandram, V., \& White, L. (2006). Switching barriers in business-to-business services: a qualitative study. International Journal of Service Industry Management, 17(2), pp. 158-192.

Zeithaml, V. A. (1988). Consumer perceptions of price, quality, and value: a means-end model and synthesis of evidence. The Journal of Marketing, 52 , pp. 2-22.

Zeithaml, V. A., Berry, L. L., \& Parasuraman, A. (1996). The behavioral consequences of service quality. the Journal of Marketing, pp. 31-46. 